\title{
Degeneración corticobasal: reporte de un caso
}

\section{Corticobasal Degeneration: A case report}

\section{Juan Pablo Gómez R. \\ Fonoaudiólogo*}

* Alumno del Diploma en Neuropsicología y Demencias, Universidad de Chile

\footnotetext{
Contacto con el autor:

Juan Pablo Gómez La Fragua Central 5438, Peñalolén Santiago - Chile Tel: (56 9) 81836361 Correo-e: jpgomez.pizarro@gmail.com

Recibido: 03/03/2015 Aceptado: 01/10/2015
}

\section{RESUMEN}

Se presenta un caso de Degeneración Corticobasal en una paciente chilena de 48 años de edad. La evolución clínica fue progresiva, con parkinsonismo (rigidez, acinesia, alteraciones posturales, disartria), deterioro cognitivo, apraxia del vestir, apraxia constructiva e hipomimia. Además, la paciente presentaba afasia no fluente. Adicionalmente, fue diagnosticada con Síndrome Depresivo Crónico (SDC) y Trombosis Venosa Profunda (TVP) de la extremidad inferior izquierda. Se han descrito varios tipos de enfermedades neurodegenerativas en las últimas décadas, las cuales cumplen con distintas características patológicas y clínicas. Sin embargo, la diferenciación de estas, en la práctica, es compleja y se necesita de una apreciación clínica entrenada, evaluaciones clínicas detalladas, neuroimágenes y estudios de laboratorio para lograr llegar a un diagnóstico adecuado. Dentro de las principales enfermedades neurodegenerativas que se presentan con parkinsonismo encontramos las llamadas "taupatías", que son la Degeneración Corticobasal (DCB) y la Parálisis Supranuclear Progresiva (PSP).

Palabras clave: degeneración corticobasal, demencia, parkinsonismo, distonía, disartria, afasia.

\begin{abstract}
A case study of corticobasal degeneration in a Chilean 48-year-old female patient is reported. The clinical course was progressive and included Parkinsonism (rigidity, akinesia, postural abnormalities, dysarthria), cognitive impairment, dressing apraxia, constructive apraxia and hypomimia. In addition, the patient had non-fluent aphasia. Also, the patient was diagnosed with Chronic Depressive Syndrome and Deep Venous Thrombosis (DVT) of the left lower extremity. In recent decades, several types of neurodegenerative diseases have been described. These diseases have different clinical and pathologic features. However, in practice, it is difficult to differentiate them from one another and, to reach an accurate diagnosis, advanced medical skills, detailed clinical evaluations of neuroimaging and laboratory studies are needed. "Tauopathies", which are Corticobasal Degeneration (CBD) and Progressive Supranuclear Palsy (PSP) are among the major neurodegenerative diseases occurring with Parkinsonism.
\end{abstract}

Keywords: corticobasal degeneration, dementia, Parkinsonism, dystonia, dysarthria, aphasia.

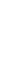




\section{Introducción}

La Degeneración Cortico Basal (DCB) se encuentra dentro del subgrupo de los parkinsonismos (Labos, Slachevsky, Fuentes y Manes, 2008). Fue definida por Rebeiz y su equipo en 1968, cuando utilizó el término “degeneración corticodentatonígrica con acromasia neuronal" para señalar los hallazgos patológicos encontrados en tres pacientes: una atrofia cortical con extensa pérdida neuronal, gliosis y neuronas edematizadas que no se teñían con la mayoría de las tinciones empleadas (acromasia). Así mismo, los pacientes presentaban un inusual compromiso motor progresivo, con lentitud y torpeza de extremidades, temblor, postura distónica, rigidez y desórdenes de la marcha, que evolucionaban a la muerte en seis $u$ ocho años (Vélez, Cosentino y Torres, 1997).

El cuadro clínico de la DCB se inicia habitualmente entre la sexta y la séptima década de vida, en forma de parkinsonismo asimétrico y dificultad para realizar labores habituales de forma desproporcionada al trastorno motor, debido a la pérdida de funciones práxicas parietales. En el curso de la enfermedad se añaden otros déficits corticales y movimientos anormales, como mioclonias o distonía. Poco frecuente, pero muy característico de esta enfermedad es el fenómeno de "mano ajena", por el que el paciente pierde la conciencia y el control del movimiento de uno de sus miembros superiores, que parece adquirir vida propia independiente. La memoria y la orientación temporoespacial no sufren un deterioro manifiesto hasta fases avanzadas de la enfermedad (Sánchez, 2010).
En cuanto a la neuropatología de la DCB, esta se encuentra dentro de las demencias corticosubcorticales (Labos et al., 2008). El depósito anormal de tau intraneuronal en la DCB son acúmulos acromásicos en las neuronas (Donoso, Miranda, Díaz, Silva y Vásquez, 2003).

La pérdida neuronal es mayor en la sustancia negra y en el córtex frontoparietal. Existe atrofia cortical mayor en frontal posterior perirolándico y parietal asimétrico, con afectación de área motora y sensitiva. Existe degeneración secundaria del tracto piramidal. Hay una intensa gliosis y fibrosis y pérdida de la arquitectura cerebral. Las neuronas pálidas grandes (acromasia) es lo más característico. La sustancia negra muestra una pérdida celular de la pars compacta moderada a severa, que afecta por igual a las bandas dorsal y ventral. Unas pocas células pigmentadas restantes contienen una inclusión levemente fibrilar, redondeada y débilmente basofílica, también presente en el locus coeruleus. La atrofia se caracteriza por una pérdida difusa de neuronas del manto cortical, además de la presencia de células de Pick. En proporción a la pérdida neuronal cortical se observa desmielinización y gliosis de la sustancia blanca subcortical subyacente. Puede objetivarse una atrofia difusa. También hay pérdida neuronal en el estriado, especialmente en el putamen, pálido, subtálamo, núcleo rojo, tálamo, tectum y tegmentum mesencefálico (Ezpeleta, 1996).

En la ecotomografía transcraneana (ECO-TC) usualmente existe hiperecogenicidad tanto de la sustancia negra como de los núcleos lenticulares, en tanto que en PSP suele existir hiperecogenicidad únicamente de los núcleos lenticulares (Venegas, Miranda y Silva, 2011). 
Respecto a las alteraciones motoras, la DCB corresponde a una enfermedad progresiva que se caracteriza por presentar desórdenes motores como parkinsonismo asimétrico, distonía, mioclonía, apraxia ideomotora, el síndrome de la mano ajena y demencia, como se mencionó antes.

El parkinsonismo asimétrico se caracteriza por presentar rigidez, bradicinesia, trastorno de la marcha y temblor, que es habitualmente más notorio en una extremidad superior. A medida que progresa la enfermedad los signos motores se vuelven más bilaterales (Méndez, 2000).

Las distonías son trastornos del movimiento en los que contracciones sostenidas del músculo causan torceduras y movimientos repetitivos o posturas anormales. Estos movimientos, que son involuntarios y a veces dolorosos, pueden afectar a un solo músculo, a un grupo de músculos (tales como los de los brazos, las piernas o el cuello) o al cuerpo entero (National Institutes of Health [NIH], 2012).

Los síntomas iniciales pueden incluir un deterioro de la escritura después de escribir varias líneas, calambres del pie y una tendencia a levantar o arrastrar un pie después de correr o de caminar una cierta distancia. El cuello puede girar o moverse involuntariamente, especialmente cuando el individuo está cansado. Otros síntomas posibles son los temblores y dificultades en la voz o en el habla. Es posible que los síntomas iniciales sean muy leves y se perciban solamente después de un esfuerzo prolongado, tensión o fatiga $(\mathrm{NIH}, 2012)$. Con el tiempo, los síntomas pueden llegar a ser más notorios, extensos y continuos. En la DCB es más común que haya distonía en brazos y de forma asimétrica, pero menos común es que se presente en cuello y tronco.

Los pacientes evidencian escasa o nula respuesta a L-dopa, incluso con dosis de hasta $1.000 \mathrm{mg} /$ día, fraccionados y administrados con el estómago vacío. Existe hipostesia de tipo cortical. Puede existir apalestesia, extinción sensitiva y falla en la discriminación de dos puntos. En etapas tardías aparece disfagia. Poseen inestabilidad postural y caídas, lo que diferencia a la DCB de la enfermad de Parkinson (Donoso, González y Behrens, 2009).

En cuanto a las alteraciones cognitivas, los pacientes con DCB presentan demencia relativamente tardía. Generalmente hay más compromiso de la función ejecutiva, la atención y el manejo del espacio (negligencia y apraxia constructiva) que de la memoria. Con menor frecuencia puede encontrarse afasia, apraxia del habla, fallas en el seguimiento ocular y apraxia de la mirada. Pueden existir trastornos del sueño, como es el caso del trastorno conductual del sueño REM (Donoso et al., 2009).

El tipo de afasia que se da en estos pacientes es de naturaleza no fluente, pero en ocasiones es difícil distinguirla, ya que además puede presentarse disartria (Donoso et al., 2003).

La demencia que muestran estos pacientes es de tipo frontal-subcortical, ya que el defecto en la función ejecutiva y los trastornos de la afectividad son más pregnantes que las fallas de la memoria, suele existir concretismo, aplanamiento o pobreza afectiva, lentificación y la tendencia a perseverar (Donoso et al., 2003).

Las alteraciones de conducta pueden ser más tardías, pero paulatinamente se establece un 
síndrome frontal, con apatía y descuido. El correlato anatómico es una atrofia de la corteza parietal y de los ganglios basales de un hemisferio. No se sabe por qué en algunos casos compromete el derecho y en otros, el izquierdo. Predomina la apatía y, en ocasiones, puede haber euforia. Puede existir anosognosia, fallas en atención y concentración, acalculia y negligencia espacial (Donoso et al., 2009).

Los pacientes con DCB pueden presentar el síndrome de Balint que consiste en un grupo de síntomas visuales caracterizado por agnosia y ataxia óptica. El paciente experimenta nistagmo, o pérdida del control de los movimientos oculares, con pérdida simultánea de la capacidad perceptiva. El paciente puede empezar a seguir un objeto en movimiento, pero luego lo pierde. La causa es una enfermedad bilateral de las áreas temporoparietales del cerebro (Robertson, 2003).

\section{Presentación del caso}

Los antecedentes han sido adquiridos a través de la ficha clínica de la paciente y de los datos de evaluación neurológica y neuropsicológica del Hospital Clínico de la Universidad de Chile.

Se trata de una paciente de sexo femenino, de nacionalidad chilena, con 48 años de edad, 12 años de estudios, con antecedentes de enfermedad de Alzheimer por parte de su abuela y antecedentes de esquizofrenia por parte de su madre. Se evalúa en marzo del 2012 donde se presenta con antecedentes de síndrome depresivo crónico, en tratamiento hace un año con Sertralina, y para la fecha con Lexapro 10, Clonazepam 0,5 mg y Aripiprazol 5 mg.

Un mes antes de la evaluación se le diagnosticó trombosis venosa profunda en la extremidad inferior izquierda, la cual ha sido tratada con Neosintrom.

Cerca de seis meses antes de la evaluación, presentó dificultades a nivel de habla y lenguaje, episodios en los que olvidaba lo que estaba diciendo y las palabras, y según la familia también tenía problemas de memoria, lo que la llevó a trabajar menos. En ocasiones presenta episodios donde confunde su imagen en el espejo y muestra dificultades para vestirse. Según su hija manifestó alucinaciones visuales. Por un episodio de angustia decide dejar de trabajar.

Al examen neurológico presentó los siguientes aspectos:

- Hipomimia.

- $\quad$ Rigidez axial.

- Bradicinesia bilateral simétrica.

- Rigidez apendicular.

- Dificultad en la marcha.

- Disartria donde destaca hipofonía.

- En cuanto al lenguaje es capaz de nominar, comprender y repetir, destacan parafasias fonéticas $y$, en menor grado, semánticas. Presenta escritura con micrografía y no es capaz de escribir una frase.

- En cuanto al test de MoCA presentó: orientación temporoespacial: $6 / 6$; codificación: $2 / 5$ y $4 / 5$; en evocación: 2/5 y 4/5. En fluidez fonética logró 10 palabras. En cuanto a las capacidades visoconstructivas no logró copiar el cubo ni hacer un reloj.

Estos antecedentes son sugerentes de parkinsonismo, por lo que es diagnosticada con deterioro cognitivo y posible DCB.

Se dejan indicaciones de suspender Aripiprazol, e iniciar con Grifoparkin, Quetiapina y Lexapro. 
Se realiza una nueva evaluación en abril del 2012, instancia en la cual presentó el examen de ECO-TC que mostró hiperecogenicidad de la sustancia negra. Presentó un estudio serológico normal, el RM de cerebro evidenció atrofia global. Normosmia y sueño normal.

La paciente mejoró desde el punto de vista motor, donde se mostró más ágil, pero se mantuvo la hipomimia, rigidez e hipocinesia, por lo que se le indica aumentar la dosis de Grifoparkin a $1.000 \mathrm{mg}$.

Se mantuvieron las alteraciones visoconstructivas, de habla y lenguaje. Se mantiene el diagnóstico de degeneración corticobasal.

\section{Comentarios finales}

La DCB es una enfermedad neurodegenerativa compleja y de difícil diagnóstico, debido a su variabilidad de presentación y superposición con otras enfermedades neurodegenerativas. Se necesitan evaluaciones específicas neuropsicológicas y neurológicas, además de una confirmación anatomopatológica. Suele confundirse con la enfermedad de Parkinson, parálisis supranuclear progresiva o demencia frontotemporal, en etapas avanzadas de la enfermedad. Se diferencia de estas enfermedades por ser un parkinsonismo asimétrico con el síndrome de la mano ajena y, en ocasiones, por presentar el síndrome de Balint, sin el compromiso de la mirada vertical característico de la PSP. No presenta mejorías con tratamiento de Ldopa.

Sugieren el diagnóstico de DCB: el parkinsonismo presente en el paciente, la rigidez apendicular, la afasia no fluente, la disartria, alteración visoconstructiva, la atrofia global de cerebro y la hiperecogenecidad de sustancia negra en ECO-TC y el poco efecto producido por el tratamiento de L-dopa.

La anatomía patológica de la DCB es característica. En la mayoría de los casos existe una atrofia frontoparietal asimétrica, con palidez de la sustancia negra. El compromiso cortical generalmente compromete a las circunvoluciones precentral, frontal inferior, poscentral alta y parietal rostral e inferior. También existe atrofia de los ganglios basales, especialmente putamen y globus pallidum interno. Actualmente se describen tres lesiones tau-positivas típicas: neurofilamentos anormalmente fosforilados en las neuronas acromáticas, placas astrocíticas tau-positivas e inclusiones tau-positivas en oligodendroglías. En cuadros de larga evolución la atrofia puede ser relativamente simétrica, a pesar de la asimetría clínica (Donoso, 2003).

El tratamiento de la enfermedad está enfocado a enlentecer el progreso de la demencia y de los trastornos motores que presenta, tratando de alivianar la carga a la familia.

El rol del fonoaudiólogo en este tipo de patología es la detección, evaluación, diagnóstico, tratamiento y educación de los adultos con desórdenes cognitivocomunicativos asociados con la demencia, además de mantener las funciones de comunicación durante la mayor cantidad de tiempo posible y entregar estrategias de comunicación para el paciente y el entorno en el cual se desarrolla, trabajo indirecto a través de modificaciones del medio ambiente, el desarrollo de rutinas, actividades terapéuticas, y la capacitación de los cuidadores.

Es importante una evaluación integral del paciente con demencia que conduzca a un 
diagnóstico diferencial adecuado, además de proporcionar información acerca de la capacidad cognitiva y comunicativa, con factores de pronóstico positivo como la habilidad para dar respuesta a señales, para leer, capacidad de seguir instrucciones simples y la competencia conversacional que demuestren la viabilidad de la intervención, ya que con ello es posible decidir si el paciente tiene el potencial de beneficiarse con la intervención, ya sea de forma directa o indirecta (Hernández, 2010).

A medida que evoluciona la enfermedad, pueden aparecer otras dificultades a nivel motor como la disfagia, por lo que es importante comenzar a trabajar la función deglutoria del paciente apenas se detectan dificultades de alimentación. La prevención y el tratamiento de la disfagia están enfocados en estrategias de compensación como cambios posturales, la consistencia del alimento, el volumen del bolo, maniobras deglutorias y manejo ambiental; y estrategias de reactivación como la estimulación termal, programa de ejercicios musculares de la cavidad orofaríngea, control y manipulación del bolo alimenticio.

\section{Referencias}

Donoso, A., González, R., y Behrens, M. (2009). El espectro de las demencias frontotemporales. Revista Hospital Clínico Universidad de Chile, 20, 231-8.

Donoso, A., Miranda, M., Díaz, M., Silva, C., y Vásquez, C. (2003). Degeneración corticobasal: 5 casos clínicos. Revista Chilena de Neuro-psiquiatría, 41(3), 191-200. http://dx.doi.org/10.4067/S0717-92272003000300004

Ezpeleta, D. (1996). Neuropatología de la enfermedad de Parkinson y los parkinsonismos. Recuperado de http://infodoctor.org/neuro/Art14.htm/

Hernández, J. (2010). Demencias: los problemas del lenguaje como hallazgos tempranos. Acta Neurológica Colombiana, 26, 3(1), 101-111.

Labos, E., Slachevsky, A., Fuentes, P., y Manes, F. (2008). Tratado de neuropsicología clínica. Buenos Aires: Akadia.

Méndez, M. (2000). Corticobasal ganglionic degeneration with Balint's syndrome. The Journal of Neuropsychiatry and Clinical Neurosciences, 12, 273-275.

National Institutes of Health, NIH. (2012). Distonía. Recuperado de http://www.nlm.nih.gov/medlineplus/spanish/dystonia.ht $\mathrm{ml} /$

Robertson, L. (2003). Binding, spatial attention and perceptual awareness, Balint's syndrome. Nature Reviews Neuroscience, $\quad 4, \quad 93-102$. http://dx.doi.org/10.1038/nrn1030

Sánchez, J. (2010). Agnosia visual. Ciencia \& Tecnología para la Salud Visual y Ocular 8(1), 115-128.

Vélez, M., Cosentino, C., y Torres, L. (1997). Degeneración corticobasal: reporte de dos casos. Revista Peruana de Neurología, 3(1-3), 19-22.

Venegas, P., Miranda, G., y Silva, C. (2011). Estudio de correlación clínico-radiológica, sensibilidad y especificidad de ecotomografía transcraneana en enfermedad de Parkinson y otros trastornos del movimiento. Revista Médica de Chile, 131(1), 54-59. 